\title{
Pseudomonas aeruginosa in Otitis Media: Prevalence and Antimicrobial Susceptibility Pattern in a Tertiary Care Hospital in India
}

\author{
Swati Mittal, Ravi Kumar Tiwary*, Amandeep Kaur, Rishabh Garg, \\ Sonia Mittal and Arup Pegu
}

Department of Microbiology, Adesh Medical College \& Hospital, NH-1, Vill-Mohri, TehsilShahbad (M), Dist-Kurukshetra, Haryana-136135, India

*Corresponding author

\section{A B S T R A C T}

\begin{tabular}{|l||}
\hline Ke y w o r d s \\
Otitis media, \\
Pseudomonas \\
aeruginosa, \\
Antimicrobial \\
susceptibility \\
testing, Kirby Bauer \\
Disk diffusion, \\
Antimicrobial \\
resistance \\
\hline Article Info \\
\hline $\begin{array}{l}\text { Accepted: } \\
\text { 18 April } 2020 \\
\text { Available Online: } \\
\text { 10 May } 2020\end{array}$ \\
\hline
\end{tabular}

Otitis media is an inflammation of the middle ear and mastoid cavity, with recurrent ear discharge through a perforated tympanic membrane. Pseudomonas aeruginosa has been particularly blamed for deep seated and progressive destruction of middle ear structures through its toxins and enzymes. The present study was done to determine the prevalence and antibiotic susceptibility of Pseudomonas aeruginosa isolated from otitis media. Patients fulfilling clinical criteria were considered into the study. Two ear swabs were taken from each patient. Sample processing and identification of organisms was done by standard bacteriological methods as per CLSI guidelines. Antimicrobial susceptibility was done by Kirby Bauer Disk diffusion method with 12 different antibiotics for Pseudomonas aeruginosa strains. Out of 131 samples, 110 (83.97\%) has shown growth of significant pathogens. Pseudomonas aeruginosa strains were isolated from 45 (38.46\%) samples. The resultsof antimicrobial susceptibility testing showed maximum sensitivity to Colistin, Imipenem and Polymyxin B (100\%), followed by Aztreonam (95.56\%), PiperacillinTazobactam (93.33\%), Amikacin, Ceftazidime, Cefipime, Cefaperazone-Sulbactam (84.44\%), Ciprofloxacin (82.22\%), Gentamicin (80\%) and Levofloxacin (68.89\%). Knowing the etiological agents of otitis media and their antibiotic susceptibility is of paramount importance for an efficient treatment and prevention of both disease complications and antimicrobial resistance.

\section{Introduction}

Otitis media (OM) refers to a group of complex infections and inflammatory diseases affecting the middle ear. While OM occurs with different degrees of severity, it invariably presents a serious healthcare problem, with significant medical, psychological and social impacts. As quoted by Hippocrates "Acute pain of the ear, with the continued fever, is to be dreaded, for there is danger that the man may become delirious and die" (Slatter, 2003).

Typical pathogens reach the middle ear either through insufflations of respiratory pathogens through the Eustachian tube from the Nasopharynx, orspread from the external ear 
canal inwards through a non-intact tympanic membrane (Verhoeff, 2006).

Bacteria, fungi and viruses are all potential pathogens in Otitis media. Knowledge of the true frequency of polymicrobial infection, particularly the extent of anaerobic involvement, is limited by differences in collection and culture techniques (Brook et al., 1992).

Bacteria commonly found responsible for causing Otitis media include: Aerobic or facultative anaerobic organisms (e.g. Pseudomonas aeruginosa, Escherichia coli, Staphylococcus aureus, Streptococcus pyogenes, Proteus mirabilis, Klebsiella pneumoniae) and anaerobic organisms, (e.g. Bacteriodes, Peptostreptococcus, Propionibacterium) (Barobby, 1988). These bacteria are occasionally found as a normal flora on the skin of the external ear canal, but may flourish due to trauma, inflammation, lacerations or high humidity. Thus eventually may then gain entry to the middle ear cleft through a chronic perforation (Mawson et al., 1988).

Pseudomonas aeruginosa is one of the predominant offending organisms causing Otitis media. The organism acts as an opportunistic pathogen, flourishes in external auditory canal and causes suppurative disease. Considering various findings, it is evident that bacterial predominance and antibiotic susceptibility pattern changing over time, makes continuous and periodic surveillance necessary in guiding appropriate antibacterial therapy.

Since Otitis Media can cause significant morbidity, knowledge of the pathogens for Otitis Media and their antibiotic susceptibility pattern helps in the appropriate usage of antibiotics that can minimize the complications.

\section{Materials and Methods}

Present study was a cross sectional study conducted in the Department of Microbiology and ENT, AIMSR, Bathinda, from November 2016 to October 2017. All the patients fulfilling both clinical and microbiological criteria for Otitis Media were considered into the study. The outcomes were studied by using percentages and frequencies.

Single use mini-tip sterile cotton swabs were used for sample collection and were transported in peptone water to maintain the swabs moist until being analyzed. All specimens were processed within 1 hour of collection. Ear discharge was collected under strict aseptic precautions in clinically diagnosed cases of otitis media. Excess discharge was mopped out from external auditory canal. Two swabs from each patient were procured from the ear discharge by using sterile cotton swabs in the tympanic cavity (Collee et al., 2006).

Sample processing and identification of organisms was done by standard bacteriological methods as per CLSI guidelines (Collee et al., 2006). The first swab was processed for direct microscopy by Gram staining and $\mathrm{KOH}$ mount, for the presence of pus cells, epithelial cells, bacteria and yeast. The second swab was cultured aerobically on blood agar and MacConkey agar, and then incubated at $37^{\circ} \mathrm{C}$ for 24 to 48 hours.

Isolates were identified from colony characters on Blood agar medium, MacConkey agar medium and any special media used for growth. Organisms were further confirmed on the basis of various biochemical tests and other special tests required for that particular organism.

Identification of Pseudomonas aeruginosa 
was based on following criteria:

On Blood agar medium: - It produced $\beta$ haemolytic, grey, moist, irregular, low convex colonies with typical fruity odor.

On MacConkey agar medium: - It produced pale non- lactose fermenting (NLF) colonies.

To see pigment production in a good manner by Pseudomonas aeruginosa, subculture was made on Nutrient agar medium: - It produced large, opaque, irregular colonies with metallic sheen and blue green diffusible pigment, which had a characteristic sweet ether or alcohol-like fruity odour (Figure 1).

On Gram staining it was slender Gramnegative bacilli, arranged singly and occasionally capsulated organism.

Pseudomonas aeruginosa was further identified by positivity of oxidase test, oxidative result on $\mathrm{O} / \mathrm{F}$ test, Catalase test, NR test, Citrate test, TSI with $\mathrm{K} / \mathrm{K}$ reaction without $\mathrm{H}_{2} \mathrm{~S}$, motility and showed growth at $42^{\circ} \mathrm{C}$ (Figure 2).

Antimicrobial susceptibility was done by Kirby Bauer Disk diffusion method with 12 different antibiotics for Pseudomonas aeruginosa strains. Antimicrobials susceptibility testing was performed on Mueller Hinton Agar (MHA) by Kirby Bauer disc diffusion method as per CLSI guidelines (CLSI, 12 ${ }^{\text {th }}$ edition, 2015).

Plates of Muller Hinton agar after drying was inoculated by lawn culture method using sterile swab that had been submerged in a bacterial suspension (standardized to match turbidity of the $0.5 \times 10^{8} \mathrm{CFU} / \mathrm{ml}$ ). Within 15 minutes of inoculation, the antimicrobial discs were applied over the plate and the plates were incubated for $16-18$ hours at $37^{\circ} \mathrm{C}$. The Antibiotics used were: Amikacin $(30 \mathrm{mcg}$ ),
Aztreonam (30 mcg), Ceftazidime (30 mcg), Cefaperazone + Sulbactam (75/30 mcg), Cefipime (30 mcg), Ciprofloxacin $(5 \mathrm{mcg})$, Colistin (10 mcg), Gentamicin (10 mcg), Imipenem (10 mcg), Levofloxacin $(5 \mathrm{mcg})$, Piperacillin + Tazobactam $(100 / 10 \mathrm{mcg})$, and Polymyxin B (300 mcg). The zone of inhibition was measured around each disc and interpretation made according to standard charts (Figure 3).

\section{Results and Discussion}

In the present study, out of 131 samples collected from otitis media patients, 110 (83.97\%) specimens were positive for culture and $21(16.03 \%)$ specimens were sterile for growth. A total of 117 isolates were obtained from 110 culture positive samples.

On gram staining, out of 117 isolates, $71(60.68 \%)$ isolates were Gram-negative organisms and 46(39.32\%) isolates were Gram-positive organisms. Maximum number of organism isolated was Pseudomonas aeruginosa, 45 (38.46\%), followed by Staphylococcus aureus 39 (33.33\%), Escherichia coli 14 (11.97\%), Klebsiella pneumoniae 12 (10.26\%), Coagulase negative Staphylococcus $4(3.42 \%)$ and Enterococcus faecalis3 (2.56\%) (Figure 4).

Pseudomonas aeruginosa showed maximum sensitivity to Colistin, Imipenem and Polymyxin B (100\%), followed by Aztreonam (95.56\%), Piperacillin-Tazobactam (93.33\%), Amikacin, Ceftazidime, Cefipime, Cefaperazone-Sulbactam (84.44\%), moderate sensitivity to Ciprofloxacin (82.22\%) and Gentamicin (80\%). Maximum resistance was observed to Levofloxacin (31.11\%) (Table 1). 
Table.1 Antibiotic susceptibility pattern of Pseudomonas aeruginosa isolates

\begin{tabular}{|l|c|c|c|c|}
\hline \multirow{2}{*}{ Antibiotic } & \multicolumn{2}{|c|}{ Number } & \multicolumn{2}{c|}{ Percentage (\%) } \\
\cline { 2 - 5 } & Sensitive & Resistant & Sensitive & Resistant \\
\hline Amikacin & 38 & 7 & 84.44 & 15.56 \\
\hline Aztreonam & 43 & 2 & 95.56 & 4.44 \\
\hline Ceftazidime & 38 & 7 & 84.44 & 15.56 \\
\hline Cefapime & 38 & 7 & 84.44 & 15.56 \\
\hline Ciprofloxacin & 38 & 7 & 84.44 & 15.56 \\
\hline Colistin & 37 & 8 & 82.22 & 17.78 \\
\hline Gentamicin & 45 & 0 & 100 & 0 \\
\hline Imipenem & 36 & 9 & 80 & 20 \\
\hline Levofloxacin & 45 & 0 & 100 & 0 \\
\hline Piperacillin-Tazobactam & 31 & 14 & 68.89 & 31.11 \\
\hline Polymyxin B & 42 & 3 & 93.33 & 6.67 \\
\hline Total & 45 & 0 & 100 & 0 \\
\hline
\end{tabular}

Figure.1 Pigment producing Pseudomonas aeruginosa on nutrient agar medium

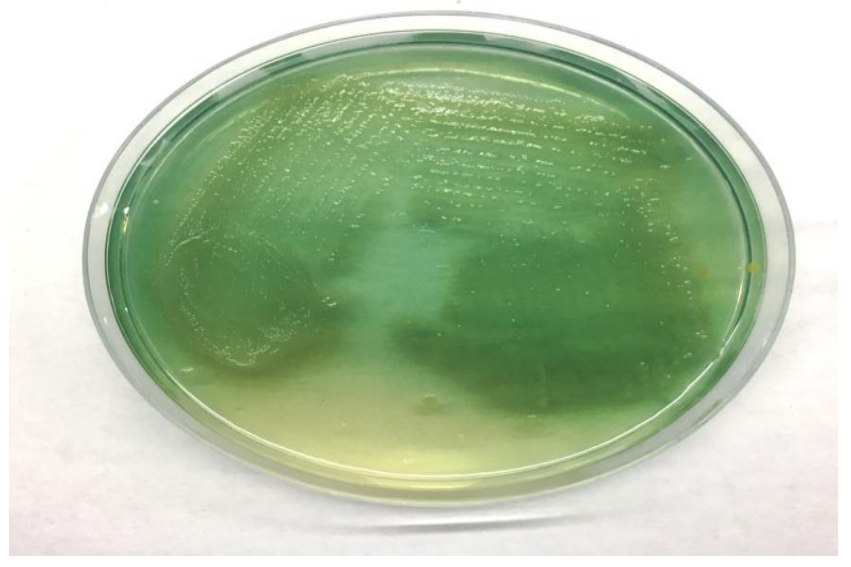

Figure.2 Biochemical reactions of Pseudomonas aeruginosa

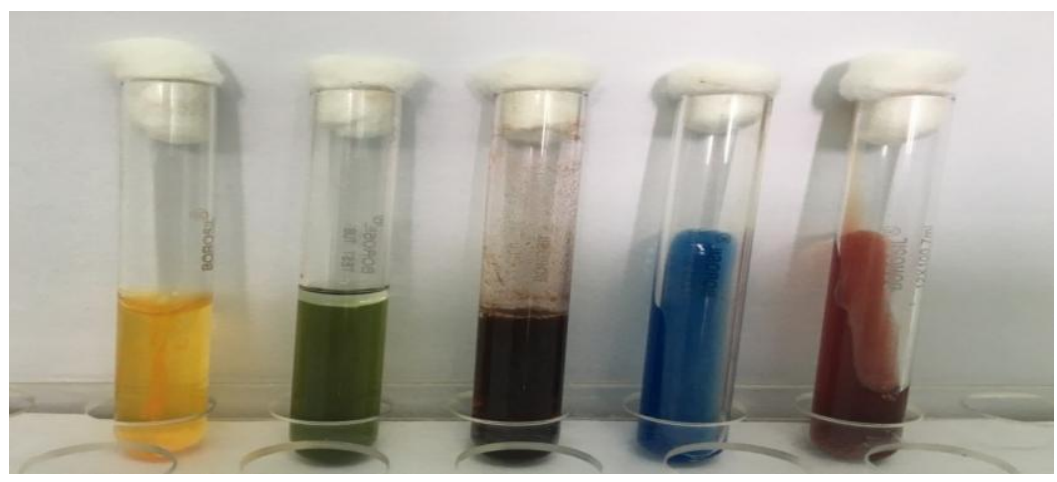

$\mathrm{O} / \mathrm{F}$ test - Oxidative $(1,2), \mathrm{NR}+(3)$, Citrate $+(4), \mathrm{TSI}-\mathrm{K} / \mathrm{K}$ without $\mathrm{H}_{2} \mathrm{~S}(5)$ 
Figure.3 Antibiotic sensitivity testing of Pseudomonas aeruginosa

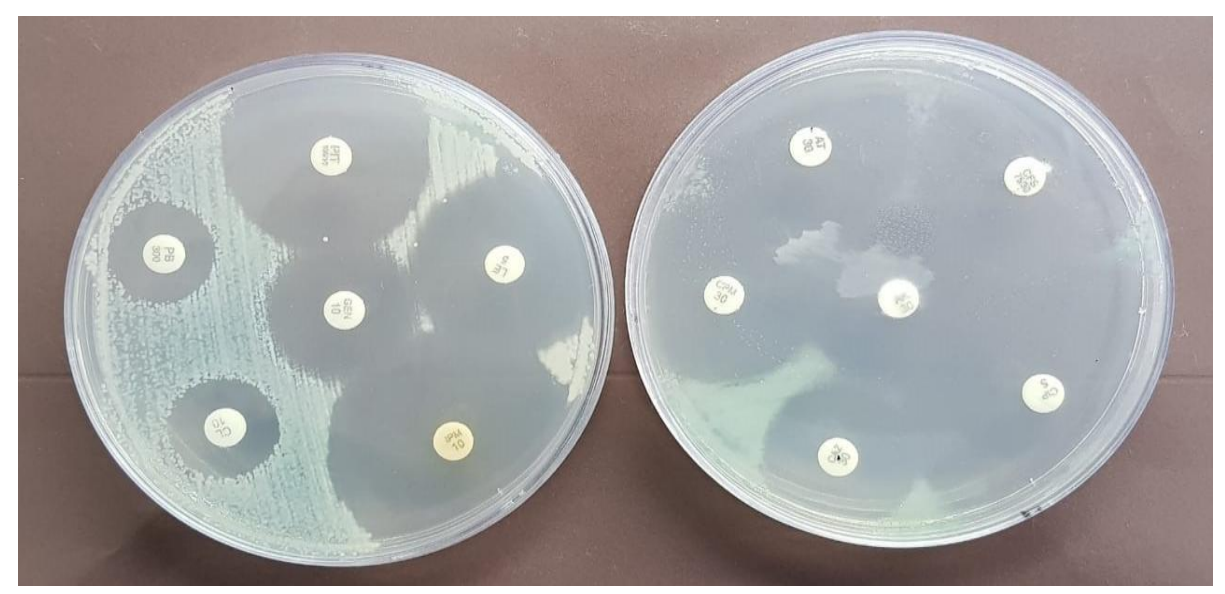

Figure.4 Bacteriological profile

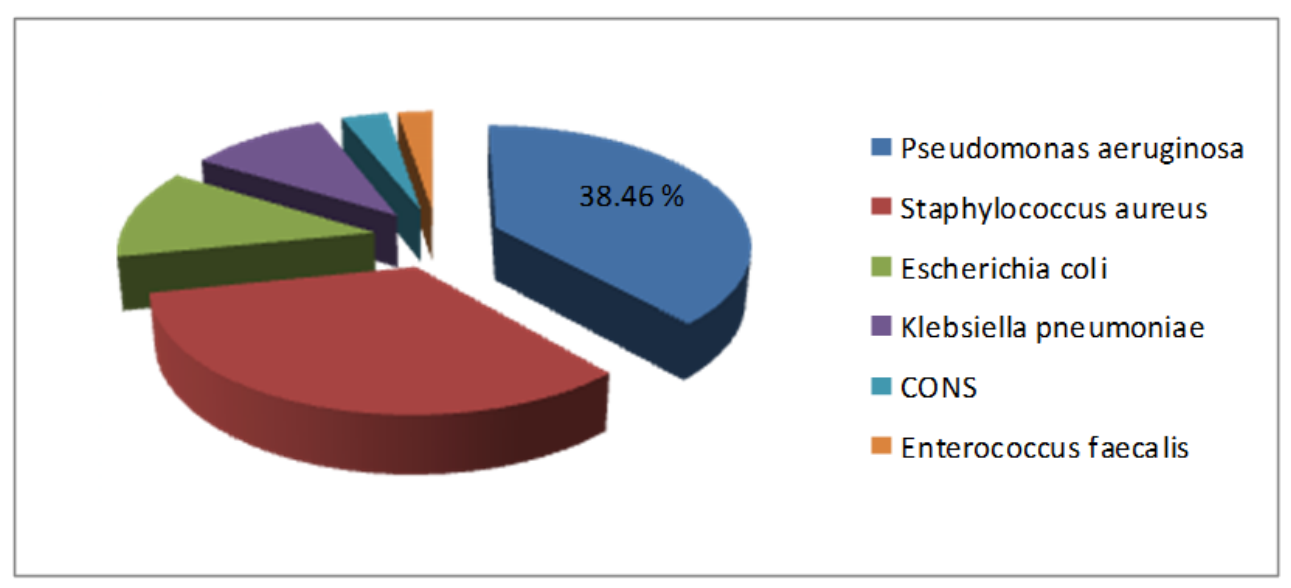

Otitis media $(\mathrm{OM})$ is an inflammatory disease of middle ear mucosa. Otitis media of varying degree is a very commonly observed condition, particularly in a developing country especially among low socioeconomic society. Its significance lies in the dreaded complications and chronicity associated with the disease process. Both Gram positive and Gram-negative organisms are responsible for middle ear infections.

In the present study, analysis of the total of 131 specimens revealed that $110(83.97 \%)$ were culture positive and $21(16.03 \%)$ were culture sterile. The culture results correlates with other authors like Wadile et al., (2015), and Aliyu et al., (2017). Sterile cultures can be attributed to anaerobic bacteria, nonbacterial organisms, presence of antimicrobial enzymes i.e. lysozyme alone or in combination with immunoglobulins that suppress the bacterial growth.

Gram negative organisms accounted for $71(60.68 \%)$ and Gram positive organisms were $46(39.32 \%)$. It is mostly seen that Gram negative organisms outnumber Gram positive organisms in otitis media as reported by Wadile et al., (2015) and Ghosh et al., (2015), which is comparable to present findings. Gram-negative organisms inhabit human gut and body as saprophytes, are found to be 
transferred to ear mostly through environmental and personal unhygienic practices. The higher incidence of Gramnegative organisms in our study could be because of fecal contamination of water, which occur during bathing or swimming.

In the present study, 6 bacterial genera were isolated in 110 positive cultures. The most common organism isolated was Pseudomonas aeruginosa 45 (38.46\%) followed by Staphylococcus aureus 39 (33.33\%), Escherichia coli 14 (11.97\%), Klebsiella pneumoniae 12 (10.26\%), Coagulase negative Staphylococcus 4 (3.42\%) and Enterococcus faecalis 3 (2.56\%). Pseudomonas aeruginosa was the predominant organism isolated in studies reported by Shyamala et al., (2012), Shetty et al., (2014) andHaneefa et al., (2015), which correlates at par with our series. The occurrence of Pseudomonas aeruginosa as the predominant offending organism could be attributed to several factors. Pseudomonas aeruginosa survives competition with other pathogens could be due to minimum nutritional requirements, its relative resistance to antibiotics and its armamentarium of antibacterial products i.e., pyocyanin and bacteriocin. Apart from the above said reasons, it uses the pili to attach to the necrotic or diseased epithelium of middle ear. Once attached, the organism produces enzymes like proteases, lipopolysaccharides, etc. to elude from normal defense mechanism of the body required for fighting infections. In addition, the organism acts as an opportunistic pathogen, flourishes in external auditory canal and causes suppurative disease.

Antibiotic susceptibility testing of Pseudomonas aeruginosa isolates showed maximum sensitivity to Colistin, Imipenem and Polymyxin B (100\%), followed by Aztreonam (95.56\%), PiperacillinTazobactam (93.33\%), Amikacin, Ceftazidime, Cefipime, Cefaperazone-
Sulbactam (84.44\%), moderate sensitivity to Ciprofloxacin (82.22\%) and Gentamicin (80\%). Maximum resistance was observed to Levofloxacin $(31.11 \%)$. This is in tandem with the observations made by many other authors such as, Sahu et al., (2014), Shetty et al., (2014) and Agarwal et al., (2017).

In the modern era of antibiotics the emergence of antibiotic resistance is becoming more common due to human negligence, irrational use and self-medication of antibiotics. Moreover, patients stop taking antibiotics before completion of therapy as and when symptoms subside, thus allowing partially resistant microbes to flourish. Such practice should be discouraged strongly and patients should be educated to avoid the same. So it becomes very important to study bacteriology of Otitis Media to formulate local antibiotic policy for appropriate use of antibiotics. This will certainly help in reducing the complications associated with otitis media and to control the development of resistance among organisms to prevalent antibiotics.

\section{References}

Agarwal M, Gupta N, Pandey G. Bacterial etiology of otitis media and their antibiotic susceptibility pattern among patients coming to a tertiary care hospital, Jaipur, India. Journal of Mahatma Gandhi University of medical sciences and technology. 2017; 2(1): 79.

Aliyu IA, Kumurya AS, Bala JA, John OC. Bacteriology of otitis media and its host - environmental - infection factors. Asia pacific environmental and occupational health journal. 2017; 3(1): 20-7.

Barobby GW, Zadik P. Bacteriology of otitis media in Ghana. Tropical Doctor. 1987; 17: 91-2. 
Brook I, Burke P. The management of acute, serous and COM: The role of anaerobic bacteria. J Hosp Infect. 1992; 22: 75.

Collee JG, Miles RS, Watt B. Tests for Identification of Bacteria. In: Collee JG, Fraser AG, Marmion BP, Simmons A (Editors). Mackie \&McCartney Practical Medical Microbiology. 14th edition, Edinburgh, UK: Churchill Livingstone. 2006. Pp. 131-47.

Ghosh A, Rana A, Prasad S. Risk factors and microbiology of chronic suppurative otois media and its clinical significance in a tertiary care setup in Western Uttar Pradesh, India. International Journal of Current Medical and Applied Sciences. 2015; 6(3): 177-83.

Haneefa S, Raveendran G, Theodore RJ. A prospective study on aetiological agents of acute and chronic suppurative otitis media. J AcadClinMicrobiol. 2015; 17: 25-8.

Jean B, Franklin R, Patricia A, George M, Janet A, Stephen G, et al., Performance standards for antimicrobial disks susceptibility tests. In: Wayne PA (Editor). Approved standards M02-A11 Clinical Laboratories Standard Institute document, $12^{\text {th }}$ edition. United States of America: Clinical Laboratories Standard Institute; 2015. Pp. 7-49.

Mawson S, Pollack M. Special role of Pseudomonas aeruginosa in chronic otitis media. Ann Otol Rhinol Laryngol Head and Neck Surg. 1988; 97 (9): 10-
3.

Sahu SK, Narasimham MV, Mohanty I, Padhi S, Panda P, Parida B. Microbiological profile of chronic suppurative otitis media and in vitro antibiotic sensitivity pattern in a tertiary care hospital. Otolaryngology Online Journal. 2014; 4(4): 32-8.

Shetty AK, Shetty A. Aerobic bacteriological profile and their antibiotic susceptibility in Chronic Suppurative Otitis Media in patients from Mangalore, Karnataka State. Journal of the Academy of Clinical Microbiologist. 2014; 16(1): 632-45.

Shyamala R, Reddy PS. The study of bacteriological agents of chronic suppurative otitis media - Aerobic culture and evaluation. J. Microbiol. Biotech. Res. 2012; 2 (1):152-62.

Slatter WH. Pathology and clinical course of inflammatory disease of the middle ear. In: Decker BC (Editor). Surgery of the ear Glasscock-Shambaugh. 5th edition. Hamilton: Elsevier Ontario; 2003. Pp. 422-33.

Verhoeff M. Chronic otitis media: a review. Int J PediatrOtorhinol. 2006: 70-1.

Wadile GR, Madhusudan BV, Tukaram KV. Bacteriological Profile of Chronic Suppurative Otitis Media. Int.J.Curr.Microbiol.App.Sci. 2015; 4(6): 41-7.

\section{How to cite this article:}

Swati Mittal, Ravi Kumar Tiwary, Amandeep Kaur, Rishabh Garg, Sonia Mittal and Arup Pegu. 2020. Pseudomonas aeruginosa in Otitis Media: Prevalence and Antimicrobial Susceptibility Pattern in a Tertiary Care Hospital in India. Int.J.Curr.Microbiol.App.Sci. 9(05): 2299-2305. doi: https://doi.org/10.20546/ijcmas.2020.905.262 